\title{
THERMOELASTIC WAVES IN AN INFINITE SOLID CAUSED BY A LINE HEAT SOURCE
}

\author{
RANJIT S. DHALIWAL, SAMIR R. MAJUMDAR and JUN WANG \\ Department of Mathematics and Statistics \\ University of Calgary \\ Calgary, Alberta, Canada T2N 1N4
}

(Received October 19, 1994 and in revised form March 15, 1995)

\begin{abstract}
The generalized thermoelasticity theory recently developed by Green and Naghdi is employed to investigate thermoelastic interactions caused by a continuous line heat source in a homogeneous isotropic unbounded solid. Hankel-Laplace transform technique is used to solve the problem. Explicit expressions, for stress and temperature fields, are obtained for small time approximation. Numerical values are displayed graphically. Our results show that this theory predicts an infinite speed for heat propagation in general, and includes the second sound phenomena as a special case.
\end{abstract}

KEY WORDS AND PHRASES. Generalized thermoelasticity, thermoelastic waves. 1992 AMS SUBJECT CLASSIFICATION CODES. 73, 80

\section{INTRODUCTION.}

In recent years many papers have been devoted to the development of the generalized theory of thermoelasticity, which predicts a finite speed for heat propagation. Lord and Shulman [1], based on a modified Fourier's law, developed a generalized theory of thermoelasticity whose governing system of equations are entirely hyperbolic and hence predict finite speed for heat propagation. Green and Lindsay [2], based on an entropy production inequality proposed by Green and Laws [3], developed a temperature-rate dependent thermoelasticity that includes the temperature-rate among constitutive variables and also predicts a finite speed for heat propagation. The applications of these theories have been examined extensively by many authors (see [4]-[9]).

Recently Green and Naghdi [10] re-examined the basic postulates of thermechanics and postulated three type of constitutive repose functions for the thermal phenomena. The nature of these three types of constitutive equations is [11] such that when the respective theories are linearized, type I theory is the same as the classical heat conduction theory (based on Fourier's law); type II theory predicts a finite speed for heat propagation and involves no energy dissipation; type III theory permits propagation of thermal signals at both infinite and finite speeds [12] and there is structural difference between these field equations and those developed in [1] and [2]. 
The aim of the present paper is to study the thermoelastic interactions caused by a continuous line heat source in a homogeneous and isotropic infinite solid by employing the above mentioned type III theory. We use Hankel-Laplace transform to find the explicit expressions for stress and temperature fields in small time intervals. Our results indicate that this theory generally predicts diffusion type of heat propagation and includes the wave type of heat propagation as a special case. The counterparts of this problem in the context of theories developed in [1] and [2] have been studied respectively by Sherief and Anwar [8] and Chandrasekharaiah and Murthy [9]. Due to the structural difference between these theories, our results differ from those obtained in [8] and [9].

\section{PRELIMINARIES}

We consider a homogeneous isotropic elastic solid occupying the whole space. The governing system of equations in thermoelasticity of type III developed in [12] are

$$
\begin{gathered}
(\lambda+\mu) \mathrm{u}_{\mathrm{j},{ }_{\mathrm{ij}}}+\mu \mathrm{u}_{\mathrm{i}, \mathrm{jj}}-\gamma \theta_{, \mathrm{i}}+\rho f_{\mathrm{i}}=\rho \overline{\mathrm{u}}_{\mathrm{i}}, \\
\rho c \bar{\theta}+\gamma \theta_{,} \ddot{\mathrm{u}}_{\mathrm{i}, \mathrm{i}}=\rho \dot{\mathrm{Q}}+k \dot{\theta}_{, \mathrm{ii}}+k^{*} \theta_{, \mathrm{ij}}, \\
\sigma_{\mathrm{ij}}=\lambda \mathrm{u}_{\mathrm{k}, \mathrm{k}} \delta_{\mathrm{ij}}+\mu\left(\mathrm{u}_{\mathrm{i}, \mathrm{j}}+\mathrm{u}_{\mathrm{j}, \mathrm{i}}\right)-\gamma \delta_{\mathrm{ij}} \theta
\end{gathered}
$$

where $\lambda$ and $\mu$ are Lame constants, $\gamma=\frac{1}{3} E \beta^{*} /(1-2 \nu), E$ is Young's modulus, $\nu$ is the Poisson ratio, $\beta^{*}$ is the coefficient of volume expansion, $u_{i}$ are the components of the displacement vector, $\rho$ is the mass density, $\theta$ is the temperature deviation above the initial temperature $\theta_{0}, \sigma_{\mathrm{ij}}$ are the compomemts of stree tensor, $k$ is the thermal conductivity, $k^{*}$ is a constant, $c$ is the specific heat for processes with invariant strain tensor, $Q$ and $f_{i}$ are, respectively, the heat source and the components of the body force, measured per unit volume.

In above equations, the notation of Cartesian tensor is employed, superposed dots denote the time derivatives and a comma followed by the index $i$ denotes the partial derivative with respect to $x_{\mathbf{i}}$. Using the nondimensional variables

$$
\begin{aligned}
& x_{\mathrm{i}}^{\prime}=x_{\mathrm{i}} / \mathrm{h}, \quad t^{\prime}=t_{0} / l, \quad \theta^{\prime}=\theta / \theta_{0}, \quad \mathbf{u}_{\mathrm{i}}^{\prime}=\mathrm{u}_{\mathrm{i}} / l, \\
& \sigma_{\mathrm{ij}}^{\prime}=\sigma_{\mathrm{ij}} / \mu, \quad \rho^{\prime}=\rho / \rho_{0}, \quad f_{\mathrm{i}}=f_{\mathrm{i}} l / a_{0}^{2}, \quad \mathrm{Q}^{\prime}=\mathrm{Q} l / a_{0}^{3},
\end{aligned}
$$

where $l$ is a standard length, $a_{0}$ is a standard speed, $\rho_{0}$ is a standard mass density, the basic equations (2.1)-(2.3) reduce to the following (dropping primes for convenience):

$$
\begin{aligned}
& \rho \alpha_{1} \bar{u}_{\mathrm{i}}=\alpha_{2} \mathrm{u}_{\mathrm{j}}, \mathrm{ij}_{\mathrm{j}}+\mathrm{u}_{\mathrm{i}, \mathrm{jj}}-\alpha_{3} \theta_{, \mathrm{i}}+\rho \alpha_{1} f_{\mathrm{i}} \text {, } \\
& \theta_{, i \mathrm{i}}+\alpha_{4} \dot{\theta}_{, \mathrm{ii}}+\rho \alpha_{5} \dot{\mathrm{Q}}=\rho \alpha_{6} \ddot{\theta}+\alpha_{7} \overline{\mathrm{u}}_{\mathrm{i}, \mathrm{i}} \text {, } \\
& \sigma_{i j}=\frac{\lambda}{\mu} u_{k, k} \delta_{i j}+\left(u_{i j}+u_{j},_{i}\right)-\alpha_{3} \theta,
\end{aligned}
$$




$$
\begin{gathered}
\alpha_{1}=\rho_{0} a_{0}^{2} / \mu, \quad \alpha_{2}=(\lambda+\mu) / \mu, \quad \alpha_{3}=\gamma \theta_{0} / \mu, \quad \alpha_{4}=k a_{0} /\left(k^{*} \eta\right) \\
\alpha_{5}=\rho_{0} a_{0}^{4} /\left(k^{*} \theta_{0}\right), \quad \alpha_{0}=\rho_{0} c a_{0}^{2} / k^{*}, \quad \alpha_{7}=\gamma a_{0}^{2} / k^{*} .
\end{gathered}
$$

\section{SOLUTION IN LAPLACE TRANSFORM DOMAIN}

In the present paper we consider an infinite solid containing a line heat source situated along the $x_{3}$-axis, that is,

$$
f_{\mathrm{i}}=0 \quad \text { and } \quad \mathrm{Q}=\frac{1}{2 \pi r} \mathrm{Q} . \delta(r) H(t)
$$

where $\delta(t)$ is the Dirac delta function, $H(t)$ is the Heaviside unit step function, $r=$ $\left(x_{1}^{2}+x_{2}^{2}\right)^{1 / 2}$ and $Q$. is a constant. The resulting thermoelastic interactions are axisymmetric in nature so that the displacement vector has only the radial component $\mathbf{u}=\mathbf{u}(r, t)$ and the stress tensor has only two components $\sigma_{\mathrm{r}}$ and $\sigma_{\phi}$ which are normal stresses in the radial' and transverse directions.

In the context of the problem considered, the regularity conditions are taken as

$$
\left(\sigma_{\mathrm{r}}, \sigma_{\phi}, \theta\right) \longrightarrow(0,0,0) \quad \text { as } \quad r \longrightarrow \infty,
$$

and the initial conditions at $t=0$ are

$$
\mathbf{u}=\dot{\mathrm{u}}=\theta=\dot{\theta}=0 .
$$

Transforming equations (2.4) $-(2.6)$ into cylindrical coordinates, with $f_{\mathrm{i}}=0$, we obtain

$$
\begin{gathered}
\rho \alpha_{1} \frac{\partial^{2} \mathrm{u}}{\partial t^{2}}=\frac{\partial}{\partial r}\left[\left(\alpha_{2}+1\right)\left(\frac{\partial \mathrm{u}}{\partial r}+\frac{\mathrm{u}}{r}\right)-\alpha_{3} \theta\right], \\
\theta, \alpha_{\mathrm{ii}}+\alpha_{4} \dot{\theta}_{\mathrm{ii}}+\rho \alpha_{5} \dot{\mathrm{Q}}=\rho \alpha_{8} \ddot{\theta}+\alpha_{7} \frac{1}{r} \frac{\partial}{\partial r}\left(r \partial^{2} \mathrm{u} / \partial t^{2}\right), \\
\sigma_{\mathrm{r}}=\left(\alpha_{2}+1\right) \frac{\partial \mathrm{u}}{\partial r}+\left(\alpha_{2}-1\right) \frac{\mathrm{u}}{r}-\alpha_{3} \theta, \\
\sigma_{\phi}=\left(\alpha_{2}-1\right) \frac{\partial \mathrm{u}}{\partial r}+\left(\alpha_{2}+1\right) \frac{\mathrm{u}}{r}-\alpha_{3} \theta .
\end{gathered}
$$

Introducing the thermoelastic potential function $\varphi$ defined by

$$
\mathbf{u}=\frac{\partial \varphi}{\partial r}
$$

equations (3.4) and (3.5) reduce to

$$
\rho \alpha_{1} \frac{\partial^{2} \varphi}{\partial t^{2}}=\left(\alpha_{2}+1\right) \nabla^{2} \varphi-\alpha_{3} \theta
$$




$$
\nabla^{2} \theta+\frac{\partial}{\partial t}\left[\alpha_{4} \nabla^{2} \theta+\rho \alpha_{5} Q\right]=\frac{\partial^{2}}{\partial t^{2}}\left[\rho \alpha_{6} \theta+\alpha_{7} \nabla^{2} \varphi\right]
$$

where $\nabla^{2}=\frac{\partial}{\partial r^{2}}+\frac{1}{r} \frac{\partial}{\partial r}$. Using equations (3.9) and (3.10), equations (3.6) and (3.7) reduce to

$$
\begin{aligned}
& \sigma_{\mathrm{r}}=\rho \alpha_{1} \frac{\partial^{2} \varphi}{\partial t^{2}}-\frac{2}{r} \frac{\partial \varphi}{\partial r} \\
& \sigma_{\phi}=\rho \alpha_{1} \frac{\partial^{2} \varphi}{\partial t^{2}}-2 \frac{\partial^{2} u}{\partial^{2} r}
\end{aligned}
$$

Elimination of $\theta$ between equations (3.9) and (3.10) gives

$$
\nabla^{4} \varphi+\alpha_{4} \frac{\partial}{\partial t}\left(\nabla^{4} \varphi\right)-b_{1} \frac{\partial^{3}}{\partial t^{3}}\left(\nabla^{2} \varphi\right)-b_{2} \frac{\partial^{2}}{\partial t^{2}}\left(\nabla^{2} \varphi\right)+b_{3} \frac{\partial^{4} \varphi}{\partial t^{4}}+b_{4} \frac{\partial Q}{\partial t}=0
$$

where

$$
\begin{aligned}
b_{1}=\rho \alpha_{1} \alpha_{4} /\left(\alpha_{2}+1\right), & b_{2}=\rho \alpha_{6}+\left(\rho \alpha_{1}+\alpha_{3} \alpha_{7}\right) /\left(\alpha_{2}+1\right), \\
b_{3}=\rho^{2} \alpha_{1} \alpha_{8} /\left(\alpha_{2}+1\right), & b_{1}=\rho \alpha_{3} \alpha_{5} /\left(\alpha_{2}+1\right) .
\end{aligned}
$$

Applying the Laplace transform, defined by

$$
\bar{g}(r, p)=\int_{0}^{\infty} g(r, t) \exp \{-p t\} d t, \quad \operatorname{Re}(p)>0,
$$

to equations (3.11)-(3.13) under the homogeneous initial conditions, with $Q$ given by (7), we find that

$$
\begin{gathered}
\bar{\sigma}_{\mathrm{s}}=\left(b_{5}^{2} p^{2}-\frac{2}{r} \frac{d}{d r}\right) \bar{\varphi}, \\
\bar{\sigma}_{\phi}=\left(b_{5}^{2} p^{2}-2 \frac{d}{d r}\right) \bar{\varphi}, \\
{\left[\left(1+\alpha_{4} p\right) \nabla^{4}-\left(b_{1} p+b_{2}\right) p^{2} \nabla^{2}+b_{3} p^{4}\right] \bar{\varphi}+\frac{A}{r} \delta(r)=0,}
\end{gathered}
$$

where $A=\frac{1}{2} b_{4} Q . / \pi$ and $b_{5}^{2}=\rho \alpha_{1}$. We may rewrite equation (3.16) in the form

$$
\left(\nabla^{2}-\lambda_{1}^{2}\right)\left(\nabla^{2}-\lambda_{2}^{2}\right) \bar{\varphi}=-\frac{A \delta(r)}{\pi\left(1+\alpha_{4} p\right)}
$$

where $\lambda_{1}^{2}$ and $\lambda_{2}^{2}$ are roots of the characteristic equation 


$$
z^{2}-\frac{b_{1} p+b_{2}}{1+\alpha_{4} p} p^{2} z+\frac{1}{1+\alpha_{4} p^{2}} b_{3} p^{4}=0
$$

Applying the Hankel transform defined by

$$
\hat{g}(\xi, p)=\int_{0}^{\infty} r J_{0}(\xi r) \bar{g}(r, p) d r
$$

where $J_{0}$ is the Bessel function of the first kind and of zero order, to equation (23), we find that

$$
\left(\xi^{2}+\lambda_{1}^{2}\right)\left(\xi^{2}+\lambda_{2}^{2}\right) \hat{\varphi}(\xi, p)=-\frac{A}{1+\alpha_{4} p}
$$

Using the inverse Hankel transform defined by

$$
\bar{g}(r, p)=\int_{0}^{\infty} \xi J_{0}(\xi r) \hat{g}(\xi, p) d \xi
$$

in equation (3.19), we obtain

$$
\bar{\varphi}(r, p)=\frac{A}{1+\alpha_{4} p}\left[K_{0}\left(\lambda_{1} r\right)-K_{0}\left(\lambda_{2} r\right)\right] /\left(\lambda_{1}^{2}-\lambda_{2}^{2}\right),
$$

where $K_{0}$ is the modified Bessel function of the second kind and of zero order.

Using the equation (3.20) and the following recurrence relations of the modified Bessel functions of the second kind

$$
\frac{\partial}{\partial r} K_{0}(r)=-K_{1}(r), \quad \text { and } \quad \frac{\partial}{\partial r}\left[r K_{1}(r)\right]=-r K_{0}(r),
$$

where $K_{1}(r)$ is the modified Bessel function of second kind and of order one, the equations (3.14) and (3.15) become

$$
\begin{gathered}
\bar{\sigma}_{\mathrm{r}}=\frac{A}{1+\alpha_{4} p} \sum_{\mathrm{i}=1}^{2}(-1)^{\mathrm{i}-1}\left[b_{5}^{2} p^{2} K_{0}\left(\lambda_{\mathrm{i}} r\right)+\frac{2}{r} \lambda_{\mathrm{i}} K_{1}\left(\lambda_{\mathrm{i}} r\right)\right] /\left(\lambda_{1}^{2}-\lambda_{2}^{2}\right), \\
\bar{\sigma}_{\phi}=\frac{A}{1+\alpha_{4} p} \sum_{\mathrm{i}=1}^{2}(-1)^{\mathrm{i}-1}\left[\left(b_{5}^{2} p^{2}-2 \lambda_{\mathrm{i}}^{2}\right) K_{0}\left(\lambda_{\mathrm{i}} r\right)-\frac{2}{r} \lambda_{\mathrm{i}} K_{1}\left(\lambda_{\mathrm{i}} r\right)\right] /\left(\lambda_{1}^{2}-\lambda_{2}^{2}\right) .
\end{gathered}
$$

Taking the Laplace transform of both sides of equation (3.9) and using the equation (26), we find that 


$$
\bar{\theta}=\frac{A}{\left(1+\alpha_{4} p\right) \alpha_{3}}\left[\left(\lambda_{1}^{2}-b_{5}^{2} p^{2}\right) K_{0}\left(\lambda_{1} r\right)-\left(\lambda_{2}^{2}-b_{5}^{2} p^{2}\right) K_{0}\left(\lambda_{2} r\right)\right] /\left(\lambda_{1}^{2}-\lambda_{2}^{2}\right) .
$$

The system of equations (3.21)-(3.23) gives rise to the solutions of stress and temperature fields in Laplace transform domain. The solutions in $(r, t)$ domain can be obtained by inverting the Laplace transform.

\section{SMALI-TIME SOLUTION IN $(r, t)$ DOMAIN}

It is a formidable task to find the inverse Laplace transform of equations (3.21)-(3.23). For this reason we have resorted to the case of small-time approximation. From equation (3.18), we find that

$$
\lambda_{1,2}=p\left(\frac{\left(b_{1} p+b_{2}\right) \pm \sqrt{\left(b_{1} p+b_{2}\right)^{2}-4 b_{3}\left(1+\alpha_{4} p\right)}}{2\left(1+\alpha_{4} p\right)}\right]^{1 / 2} .
$$

For large $p$, expanding the above equation binomially in ascending powers of $1 / p$ and retaining only necessary terms, we obtain

$$
\lambda_{1} \approx b_{10} p+b_{11}, \quad \lambda_{2} \approx b_{20} p^{1 / 2}
$$

by neglecting terms of $O\left(p^{-2}\right)$, where

$$
\begin{gathered}
b_{10}=\left(b_{1} / \alpha_{4}\right)^{1 / 2}=b_{5} /\left(\alpha_{2}+1\right)^{1 / 2}, \quad b_{20}=\left(b_{3}\right)^{1 / 2}, \\
b_{11}=\frac{1}{2}\left(b_{1} b_{2} \alpha_{4}-b_{3} \alpha_{4}^{2}-b_{1}^{2}\right) /\left(b_{1} \alpha_{4}\right)^{3 / 2}
\end{gathered}
$$

Similiarly, we have

$$
\frac{1}{\left(\lambda_{1}^{2}-\lambda_{2}^{2}\right)\left(1+\alpha_{4} p\right)}=\frac{1}{b_{1}} \frac{1}{p^{3}}+b_{0} \frac{1}{p^{4}}+O\left(p^{-5}\right)
$$

where

$$
b_{0}=\left(2 b_{3} \alpha_{4}-b_{1} b_{2}\right) / b_{1}^{2}
$$

Substituting from equations (4.2) and (4.3) into equations (3.21)-(3.23), we obtain

$$
\begin{gathered}
\bar{\sigma}_{\mathrm{r}} / A \approx b_{5}^{2}\left(\frac{1}{b_{1}} p^{-1}+b_{0} p^{-2}\right) K_{0}\left[\left(b_{10} p+b_{11}\right) r\right]+\frac{2}{r}\left[\left(b_{10} / b_{1}\right) p^{-2}+\left(b_{11} / b_{1}+b_{10} b_{0}\right) p^{-3}\right. \\
\left.+b_{11} b_{0} p^{-1}\right] K_{1}\left[\left(b_{10} p+b_{11}\right) r\right]-b_{5}^{2}\left(\frac{1}{b_{1}} p^{-1}+b_{0} p^{-2}\right) K_{0}\left[b_{20} p^{1 / 2} r\right]-\frac{2}{r} b_{20}\left(\frac{1}{b_{1}} p^{-5} / 2\right. \\
\left.+b_{0} p^{-7 / 2}\right) K_{1}\left[b_{20} p^{1 / 2} r\right]
\end{gathered}
$$




$$
\begin{gathered}
\bar{\sigma}_{\phi} / A \approx\left[\frac{b_{5}^{2}-2 b_{10}^{2}}{b_{1}} p^{-1}+\left(b_{5}^{2} b_{0}-2 b_{10}^{2} b_{0}-4 b_{10} b_{11} / b_{1}\right) p^{-2}-2\left(b_{11}^{2} / b_{1}+2 b_{10} b_{0}\right) p^{-3}\right. \\
\left.-2 b_{11}^{2} b_{0} p^{-4}\right] K_{0}\left[\left(b_{10} p+b_{11}\right) r\right]-\frac{2}{r}\left[\left(b_{10} / b_{1}\right) p^{-2}+\left(b_{11} / b_{1}+b_{10} b_{0}\right) p^{-3}+b_{11} b_{0} p^{-4}\right] \\
K_{1}\left[\left(b_{10} p+b_{11}\right) r\right]-\left[\left(b_{5}^{2} / b_{1}\right) p^{-1}+\left(b_{5}^{2} b_{0}-2 b_{20}^{2} / b_{1}\right) p^{-2}-2 b_{20}^{2} b_{0} p^{-3}\right] K_{0}\left[b_{20} p^{1 / 2} r\right] \\
+\frac{2}{r} b_{20}\left(\frac{1}{b_{1}} p^{-5} / 2+b_{0} p^{-7 / 2}\right) K_{1}\left[b_{20} p^{1 / 2} r\right] \\
\alpha_{3} \bar{\theta} / A \approx\left[\frac{b_{10}^{2}-b_{5}^{2}}{b_{1}} p^{-1}+\left(b_{10}^{2} b_{0}-b_{5}^{2} b_{0}+2 b_{10} b_{11} / b_{1}\right) p^{-2}+\left(b_{11}^{2} / b_{1}+2 b_{10} b_{11} b_{0}\right) p^{-3}\right. \\
\left.+b_{11}^{2} b_{0} p^{-4}\right] K_{0}\left[\left(b_{10} p+b_{11}\right) r\right]+\left[\left(b_{5}^{2} / b_{1}\right) p^{-1}+\left(b_{5}^{2} b_{0}-b_{20}^{2} / b_{1}\right) p^{-2}-b_{20}^{2} b_{0} p^{-3}\right] K_{0}\left[b_{20} p^{1 / 2} r\right] .
\end{gathered}
$$

From [13], we find the following inverse Laplace transforms

$$
\begin{gathered}
\mathscr{L}^{-1}\left\{p^{-\mathrm{n}}\right\}=t^{\mathrm{n}-1} / \Gamma(n), \\
\mathscr{L}^{-1}\left\{K_{0}(\alpha p)\right\}=H(t-\alpha)\left(t^{2}-\alpha^{2}\right)^{-1 / 2}, \\
\mathscr{L}^{-1}\left\{p^{-1} K_{0}(\alpha p)\right\}=H(t-\alpha) \cosh ^{-1}(t / a)
\end{gathered}
$$

where $\Gamma(n)$ is the Gamma function. Using the shift property of the Laplace transform and the expansion technique for large $p$, we obtain

$$
\begin{aligned}
\mathscr{L}^{-1}\left\{p^{-1} K_{0}\left[\left(b_{10} p+b_{11}\right) r\right]\right\} & =\exp \{-m t\} \mathscr{L}^{-1}\left\{\frac{1}{p-m} K_{0}\left(b_{10} p r\right)\right\} \\
& =\exp \{-m t\} \mathscr{L}^{-1}\left\{\left(p^{-1}+m p^{-2}+m^{2} p^{-3}\right) K_{0}\left(b_{10} p r\right)\right\}
\end{aligned}
$$

where $m=b_{11} / b_{10}$. Using the convolution theorem of Laplace transform and the formulas (4.7) and (4.8), we get

$$
\begin{aligned}
\mathscr{L}^{-1}\left\{p^{-2} K_{0}\left(b_{10} p r\right)\right\}=\mathscr{L}^{-1}\left\{p^{-2}\right\} * \mathscr{L}^{-1}\left\{K_{0}\left(b_{10} p r\right)\right\} \\
=[t / \Gamma(2)] *\left[H\left(t-b_{10} r\right)\left(t^{2}-b_{10}^{2} r^{2}\right)^{-1} / 2\right] \\
=\int_{0}^{t}(t-s) H\left(s-b_{10} r\right)\left(s^{2}-b_{10}^{2} r^{2}\right)^{-1 / 2} d s \\
=H\left(t-b_{10} r\right)\left[t \quad \cosh ^{-1}\left[t /\left(b_{10} r\right)\right]-\left(t^{2}-b_{10}^{2} r^{2}\right)^{1 / 2}\right] .
\end{aligned}
$$

Similiarly we obtain

$$
\mathscr{L}^{-1}\left\{p^{-3} K_{0}\left(b_{10} p r\right)\right\}=H\left(t-b_{10} r\right)\left[\left(\frac{1}{2} t^{2}+\frac{1}{4} b_{10}^{2} r^{2}\right) \cosh ^{-1}\left[t /\left(b_{10} r\right)\right]-\frac{3}{4} t\left(t^{2}-b_{10}^{2} r^{2}\right)^{1 / 2}\right] .
$$


Substituting from equations (4.9), (4.11) and (4.12) into equation (4.10), we obtain

$$
\begin{gathered}
\mathscr{L}^{-1}\left\{p^{-1} K_{0}\left[\left(b_{10} p+b_{11}\right) r\right]\right\}=H\left(t-b_{10} r\right) \exp \{-m t\}\left[\left(1+m t+\frac{1}{2} m^{2} t^{2}+\frac{1}{4} m^{2} b_{10}^{2} r^{2}\right) \times\right. \\
\left.\cosh ^{-1}\left(\frac{t}{b_{10}}\right)-m\left(1+\frac{3}{4} m t\right)\left(t^{2}-b_{10}^{2} r^{2}\right)^{1 / 2}\right]
\end{gathered}
$$

Applying similiar procedure to other terms and making use of the following identities

$$
\begin{gathered}
\mathscr{L}^{-1}\left\{K_{1}(\alpha p)\right\}=\frac{t}{\alpha} H(t-\alpha)\left(t^{2}-\alpha^{2}\right)^{-1 / 2} \\
\mathscr{L}^{-1}\left\{p^{-1} K_{1}(\alpha p)\right\}=\frac{1}{\alpha} H(t-\alpha)\left(t^{2}-\alpha^{2}\right)^{1 / 2} \\
\mathscr{L}^{-1}\left\{K_{0}(\sqrt{\alpha p})\right\}=\frac{1}{2 t} \exp \left\{-\frac{\alpha}{4 t}\right\} \\
\mathscr{L}^{1}\left\{p^{-1 / 2} K_{1}(\sqrt{\alpha p})\right\}=\alpha^{-1 / 2} \exp \left\{-\frac{\alpha}{4 t}\right\}
\end{gathered}
$$

we find that for small values of time

$$
\begin{aligned}
& \sigma_{\mathrm{s}} / A=H\left(t-b_{10} r\right) \exp \{-m t\}\left[\sigma_{\mathrm{r} 1} \cosh ^{-1}\left(\frac{t}{b_{10}}\right)+\sigma_{\mathrm{r} 2}\left(t^{2}-b_{10}^{2} r^{2}\right)^{1 / 2}\right]+\sigma_{\mathrm{r} 3} E_{1}\left\{b_{20}^{2} r^{2} /(4 t)\right\} \\
& +\sigma_{\mathrm{r} 4} \exp \left\{-b_{20}^{2} r^{2} /(4 t)\right\}, \\
& \sigma_{\phi} / A=H\left(t-b_{10} r\right) \exp \{-m t\}\left[\sigma_{\phi 1} \cosh ^{-1}\left(\frac{t}{b_{10} r}\right)+\sigma_{\phi 2}\left(t^{2}-b_{10}^{2} r^{2}\right)^{1 / 2}\right]+\sigma_{\phi 3} E_{1}\left\{b_{20}^{2} r^{2} /(4 t)\right\} \\
& +\sigma_{\phi 1} \exp \left\{-b_{20}^{2} r^{2} /(4 t)\right\}, \\
& \alpha_{3} \theta / A=H\left(t-b_{10} r\right) \exp \{-m t\}\left[\theta_{1} \cosh ^{-1}\left(\frac{t}{b_{10} r}\right)+\theta_{2}\left(t^{2}-b_{10}^{2} r^{2}\right)^{1 / 2}\right]+\theta_{3} E_{1}\left\{b_{20}^{2} r^{2} /(4 t)\right\} \\
& +\theta_{1} \exp \left\{-b_{20}^{2} r^{2} /(4 t)\right\}
\end{aligned}
$$

where $E_{1}\{x\}=\int_{x}^{\infty} \frac{1}{s} \exp \{-s\} d s, x>0$, is the exponential integral and

$$
\begin{gathered}
\sigma_{\mathrm{r} 1}=\frac{1}{8 b_{1}}\left[8 b_{5}^{2}+4 b_{5}^{2} b_{11}^{2} r^{2}+4 b_{0} b_{1} b_{5}^{2} b_{10} b_{11} r^{2}+3 b_{10} b_{11}-4 b_{1} b_{10}^{2}+b_{0} b_{1} b_{10}^{2}\right. \\
\left.+b_{0} b_{1} b_{10}^{3} b_{11} r^{2}\right]+\frac{t}{16 b_{1}}\left[16 b_{5}^{2} m+16 b_{0} b_{1} b_{5}^{2}-32 b_{11}^{2}-16 b_{10} b_{11}-16 b_{0} b_{1} b_{10}^{2}\right. \\
\left.-b_{0} b_{1} b_{10} b_{11}\right]+\frac{t^{2}}{2 b_{1}}\left[m^{2} b_{5}^{2}+2 m b_{0} b_{1} b_{5}^{2}-b_{0} b_{1} b_{10} b_{11}\right] \\
\sigma_{\mathrm{r} 2}=\frac{1}{2 b_{1}}\left[b_{0} b_{1} b_{10}^{2}+3 b_{10} b_{11}-2 m b_{5}^{2}-2 b_{0} b_{1} b_{5}^{2}\right]+\frac{t}{8 b_{1}}\left[\frac{1}{r^{2}}+2 \frac{m}{r^{2}}+7 b_{0} b_{1} b_{10} b_{11}\right. \\
\left.-12 m b_{1} b_{5}^{2}-12 m b_{0} b_{1} b_{5}^{2}\right]+\frac{t^{2}}{16 b_{10} b_{1} b_{11}\left[\frac{8}{r^{2}}-\frac{1}{r} b_{0} b_{1}\right]+\frac{t^{3}}{8 b_{10} r^{2} b_{0} b_{11}}} \\
\sigma_{\mathrm{r} 3}=\frac{1}{96 b_{1}}\left[b_{0} b_{1} b_{20}^{6} r^{4}+6 b_{20}^{4} r^{2}-48 b_{5}^{2}-12 b_{0} b_{1} b_{5}^{2} b_{20}^{2} r^{2}\right]+\frac{t}{16 b_{1}}\left[4 b_{0} b_{1} b_{20}^{2}+b_{0} b_{1} b_{20}^{4} r^{2}\right. \\
\left.+8 b_{20}^{2}-8 b_{0} b_{1} b_{5}^{2}\right]
\end{gathered}
$$




$$
\begin{aligned}
& \sigma_{\mathrm{r} 4}=\frac{t}{24 b_{1}}\left[12 b_{0} b_{1} b_{5}^{2}-6 b_{20}^{2}-b_{0} b_{1} b_{20}^{4} r^{2}\right]-\frac{t^{2}}{24 b_{1}}\left[24 \frac{1}{r^{2}}+5 b_{0} b_{1} b_{20}^{2}\right]-\frac{1}{3} b_{0} t^{3} / r^{2} \\
& \sigma_{\phi 1}=\frac{1}{8 b_{1}}\left[\left(8 b_{5}^{2}-8 b_{10}^{2}-2 b_{10} b_{11}-b_{0} b_{11}-b_{0}^{2} b_{1} b_{10}\right)+4\left(b_{0} b_{1} b_{5}^{2}-2 b_{0} b_{1} b_{10}^{2}-4 b_{10} b_{11}\right) b_{10} b_{11} r\right. \\
& \left.+\left(4 b_{5}^{2} b_{11}^{2}-12 b_{10}^{2} b_{11}^{2}-8 b_{0} b_{1} b_{10}^{3}-b_{0} b_{1} b_{10}^{3} b_{11}\right) r^{2}\right]+\frac{t}{16 b_{1}}\left[\left(16 m b_{5}^{2}+16 b_{0} b_{1} b_{5}^{2}-16 b_{0} b_{1} b_{10}^{2}\right.\right. \\
& \left.\left.-48 b_{10} b_{11}+b_{0} b_{1} b_{10} b_{11}\right)-8 b_{0} b_{1} b_{10}^{2} b_{11}^{2} r^{2}\right]+\frac{t^{2}}{2 b_{1}}\left(m^{2} b_{5}^{2}+2 b_{0} b_{1} m b_{5}^{2}-14 b_{11}^{2}-4 b_{0} b_{1} b_{10}\right. \\
& \left.-3 b_{0} b_{1} b_{10} b_{11}\right)-\frac{1}{3} b_{0} b_{11}^{2} t^{3} \\
& \sigma_{\phi 2}=\frac{1}{18 b_{1}}\left[\left(36 b_{10}^{2}+27 b_{0} b_{1} b_{10}^{2}+45 b_{10} b_{11}-18 b_{5}^{2}-18 b_{0} b_{1} b_{5}^{2}\right)+16 b_{0} b_{10}^{2} b_{11}^{2} r^{2}\right] \\
& +\frac{t}{8 b_{1} b_{10}}\left[\left(24 b_{0} b_{1} b_{10}^{2} b_{11}+24 b_{0} b_{1} b_{10}^{2}+84 b_{10} b_{11}^{2}-6 m^{2} b_{5}^{2} b_{10}-12 b_{0} b_{5}^{2} b_{10} b_{11}\right)\right. \\
& \left.-7 b_{0} b_{1} b_{10}^{2} b_{11} r-\left(3 b_{11}+b_{0} b_{1} b_{10}+8 b_{10}\right) \frac{1}{r^{2}}\right]+t^{2}\left[\frac{11}{18} b_{0} b_{11}^{2}+\frac{1}{16 b_{1} b_{10}}\left(b_{0} b_{1} b_{11}\right.\right. \\
& \left.\left.-12 b_{11}-4 b_{0} b_{1} b_{10}\right) \frac{1}{r^{2}}\right]-\frac{1}{8} b_{0} b_{11} t^{3} /\left(b_{10} r^{2}\right), \\
& \sigma_{\phi 3}=\frac{1}{96 b_{1}}\left[-48 b_{5}^{2}+18 b_{20}^{4} r^{2}-12 b_{0} b_{1} b_{5}^{2} b_{20}^{2} r^{2}-b_{0} b_{1} b_{20}^{6} r^{4}\right] \\
& +\frac{t}{16 b_{1}}\left[8 b_{20}^{2}-8 b_{0} b_{1} b_{5}^{2}-4 b_{0} b_{20}^{2}-b_{0} b_{1} b_{20}^{4} r^{2}\right] \text {, } \\
& \sigma_{\phi 4}=t\left[\frac{1}{2} b_{0} b_{5}^{2}-\frac{3}{4} b_{20}^{2} / b_{1}+\frac{1}{24} b_{0} b_{20}^{4} r^{2}\right]+t^{2}\left[\left(b_{1} r^{2}\right)^{-1}+\frac{5}{24} b_{0} b_{20}^{2}\right]+\frac{1}{3} b_{0} t^{3} / r^{2} \text {, } \\
& \theta_{1}=\frac{1}{4 b_{1}}\left[4\left(b_{10}^{2}-b_{5}^{2}\right)+\left(7 b_{10}^{2} b_{11}^{2}+10 b_{0} b_{10}^{3} b_{11}-2 b_{5}^{2} b_{11}^{2}-2 b_{0} b_{1} b_{5}^{2} b_{10} b_{11}\right) r^{2}\right] \\
& +\frac{t}{4 b_{1}}\left[\left(12 b_{10} b_{11}+4 b_{0} b_{10}^{2}-4 \gamma b_{5}^{2}-4 b_{0} b_{1} b_{5}^{2}\right)+b_{0} b_{1} b_{10}^{2} b_{11}^{2} r^{2}\right] \\
& +\frac{t^{2}}{2 b_{1}}\left[7 b_{11}^{2}+4 b_{0} b_{1} b_{10} b_{11}-2 m b_{0} b_{1} b_{5}^{2}-2 m^{2} b_{5}^{2}\right]+\frac{1}{6} b_{0} b_{11}^{2} t^{3} \\
& \theta_{2}=\left(b_{0} b_{5}^{2}+m b_{5}^{2} / b_{1}-b_{0} b_{10}^{2}-3 b_{10} b_{11} / b_{1}-\frac{4}{9} b_{0} b_{10}^{2} b_{11}^{2} r^{2}\right) \\
& +t\left(\frac{3}{2} m b_{0} b_{5}^{2}+\frac{3}{4} m^{2} b_{5}^{2} / b_{1}-\frac{21}{4 b_{1}} b_{11}^{2}-3 b_{0} b_{10} b_{11}\right)-\frac{11}{36} b_{0} b_{11}^{2} t^{2} \text {, } \\
& \theta_{3}=\left(\frac{1}{2} b_{5}^{2} / b_{1}+\frac{1}{8} b_{0} b_{5}^{2} b_{20}^{2}-\frac{1}{8} b_{20}^{4} r^{2} / b_{1}-\frac{1}{128} b_{0} b_{20}^{6} r^{4}\right) \\
& +t\left(\frac{1}{2} b_{0} b_{5}^{2}-\frac{1}{2} b_{20}^{2} / b_{1}-\frac{1}{8} b_{0} b_{20}^{4} r^{2}\right)-\frac{1}{2} b_{0} b_{20}^{2} t^{2}, \\
& \theta_{4}=\frac{t}{2}\left(b_{20}^{2} / b_{1}-b_{0} b_{5}^{2}+\frac{r^{2}}{16} b_{0} b_{20}^{4}\right)+\frac{3}{8} b_{0} b_{20}^{2} t^{2} .
\end{aligned}
$$

Due to the presence of the exponential integral and the exponent functions in the expressions of equations (4.18)-(4.20), it can be seen that the effect of input (7) is felt throughout the medium instantly and hence it shows that according to this theory heat travels at an infinite speed.

5. HEAT PROPAgATION WITH A FINITE SPEED

As we have pointed out earlier, this theory generally predicts a diffusion type of heat 
propagation and includes the finite wave propagation of heat as a special case. In this section we consider this special case when $k^{*}>k$, that is, $\alpha_{4} \approx 0, b_{1} \approx 0$ and equation (4.1) becomes

$$
\lambda_{\mathrm{i}}=c_{\mathrm{i}} p, \quad i=1,2,
$$

where

$$
c_{i}=\left[\left[b_{2} \pm\left(b_{2}^{2}-4 b_{3}\right)^{1 / 2}\right] / 2\right]^{1 / 2}
$$

Using equation (5.1) in equations (3.21) $-(3.23)$, we obtain

$$
\begin{gathered}
c . \bar{\sigma}_{\mathrm{r}} / A=\sum_{\mathrm{i}=1}^{2}(-1)^{\mathrm{i}-1}\left[b_{5}^{2} K_{0}\left(c_{\mathrm{i}} p r\right)+\frac{2}{r} c_{\mathrm{i}} p^{-1} K_{1}\left(c_{\mathrm{i}} p r\right)\right], \\
c_{.} \bar{\sigma}_{\phi} / A=\sum_{\mathrm{i}=1}^{2}(-1)^{\mathrm{i}-1}\left[\left(b_{5}^{2}-2 c_{\mathrm{i}}^{2}\right) K_{0}\left(c_{\mathrm{i}} p r\right)-\frac{2}{r} c_{\mathrm{i}} p^{-1} K_{1}\left(c_{\mathrm{i}} p r\right)\right], \\
c_{0} \alpha_{3} \partial / A=\sum_{\mathrm{i}=1}^{2}(-1)^{\mathrm{i}-1}\left(c_{\mathrm{i}}^{2}-b_{5}^{2}\right) K_{0}\left(c_{\mathrm{i}} p r\right),
\end{gathered}
$$

where $c_{0}=\left(b_{2}^{2}-4 b_{3}\right)^{1 / 2}$.

Making use of identities (4.8) and (4.15), we find the solution for stress and temperature fields as

$$
\begin{gathered}
c_{0} \sigma_{\mathrm{r}} / A=\sum_{\mathrm{i}=1}^{2}(-1)^{\mathrm{i}-1} H\left(t-c_{\mathrm{i}} r\right)\left[b_{5}^{2}\left(t^{2}-c_{\mathrm{i}}^{2} r^{2}\right)^{-1 / 2}+\frac{2}{r^{2}}\left(t^{2}-c_{\mathrm{i}}^{2} r^{2}\right)^{1 / 2}\right] \\
c_{.} \sigma_{\phi} / A=\sum_{\mathrm{i}=1}^{2}(-1)^{\mathrm{i}-1} H\left(t-c_{\mathrm{i}} r\right)\left[\left(b_{5}^{2}-2 c_{\mathrm{i}}^{2}\right)\left(t^{2}-c_{\mathrm{i}}^{2} r^{2}\right)^{-1 / 2}-\frac{2}{r^{2}}\left(t^{2}-c_{\mathrm{i}}^{2} r^{2}\right)^{1 / 2}\right], \\
\text { c. } \alpha_{3} \theta / A=\sum_{\mathrm{i}=1}^{2}(-1)^{\mathrm{i}-1}\left(c_{\mathrm{i}}^{2}-b_{5}^{2}\right) H\left(t-c_{\mathrm{i}} r\right)\left(t^{2}-c_{\mathrm{i}}^{2} r^{2}\right)^{-1 / 2}
\end{gathered}
$$

In this special case, solutions (5.6)-(5.8) indicate that thermal signals propagate at a finite speed since the Heaviside unit step function appears in all terms.

\section{NUMERICAL RESULTS AND CONCLUSIONS}

The numerical values of stress and temperature fields at time $t=0.5$ have been calculated and displayed in figure 1-3 along the r-axis. To obtain these numerical values, we have taken that $b_{2}=5.2, b_{3}=3.35, b_{4}=2.25$, and $b_{5}^{2}=2.54$. In the general case, we assumed that $\alpha_{4}=3.1$ and $b_{1}=2.8$.

From Fig.1 and 2, we note that in the general case the magnitudes of the radial stress and the tangential stress decrease from $-\infty$ at $r=0$ to zero as $r$ tends to infinity without 


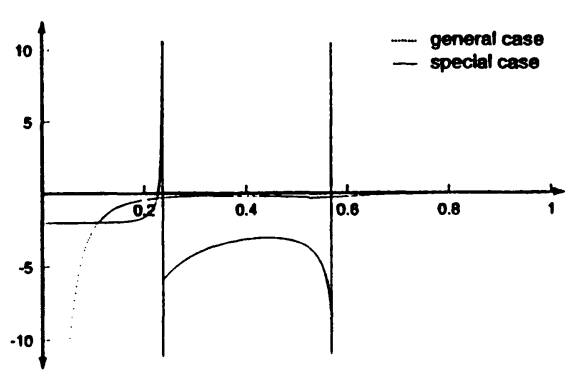

Fig.1. $\sigma_{\mathrm{r}} / A$ vs. $r$ for $t=0.5$

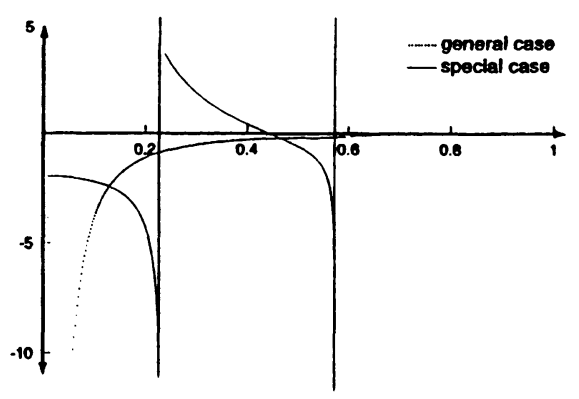

Fig.2. $\sigma_{\phi} / A$ vs. $r$ for $t=0.5$

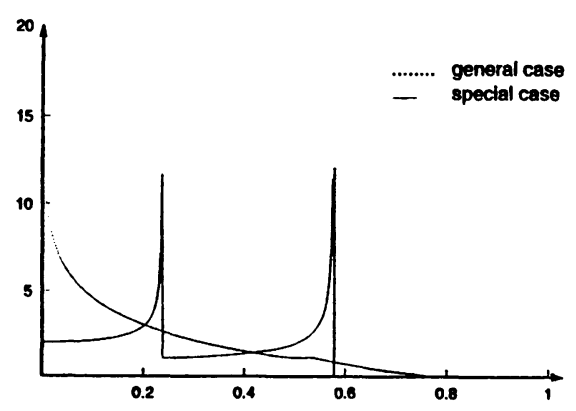

Fig.3. $\quad \alpha_{3} \theta / A$ vs. $r$ for $t=0.5$

any jumps. However, in the undamped case, the corresponding magnitudes suffer two infinite jumps at $r=t / c_{1} \approx 0.237113$ and $r=t / c_{2} \approx 0.576053$. From Fig.3, we see that in the general case the temperature decreases from to at $r=0$ to zero as $r$ tends to infinity while the temperature in the undamped case suffers two infinit jumps at $r \approx 0.237113$ and $r \approx 0.576053$ and vanishes for $r>0.576053$.

It is also apparent that, in general, this theory predicts a diffusion type of thermal propagation. The values of stress and temperature fields damp out gradually as $r$ increses. In the special case (undamped case) when $k^{*}>k$, this theory predicts finite speed for heat propagation. In this special case, stress and temperature fields vanish identically for $r>$ $t / c_{2} \approx 0.576053$.

We also note that in the special case, both the stress and the temperatue fields have finite values at $r=0$, which is quite unusual for a continuous line heat source input given by (3.1). However, if we set $k=0$ in the heat conduction equation (2.2), we obtain a hyperbolic heat equation which contains $\dot{Q}$

$$
\rho c \ddot{\theta}+\gamma \theta \cdot \ddot{\mathrm{u}}_{\mathrm{i}, \mathrm{i}}=\rho \dot{\mathrm{Q}}+k^{*} \theta_{, \mathrm{ii}}
$$

In this case we get an impulsive heat source $\dot{Q}$ when we take $Q$ as a continuous heat source given by (3.1). According to solutions (5.6) $-(5.8)$, the precise values for stress and temperature fields at $r=0$ are

$$
\sigma_{\mathrm{r}} / A=-1 / t, \quad \sigma_{\phi} / A=-1 / t, \quad \theta / A=1 / t .
$$




\section{REFERENCE}

1. H. Lord and Y. Shulman, A Generalized Dynamical Theory Of Thermoelasticity, J. Mech. Phys. Solids, 15, 299-309, 1967.

2. A. E. Green and K. A. Lindsay, Thermoelasticity, J. Elasticity, 2, 1-7, 1972.

3. A. E. Green and N. Laws, On The Entropy Production Inequality, Arch. Rat. Mech. Anal., 45, 47-53, 1972.

4. H. H. Sherief and R. S. Dhaliwal, Generalized One-dimensional Thermal-shock Problem For Small Times, J. Thermal Stresses, 4, 407-420, 1981.

5. J. Wang and R. S. Dhaliwal, Fundamental Solutions of The Generalized Thermoelastic Equations, J. Thermal Stresses, 16, 135-161, 1993.

6. R. S. Dhaliwal and J. G. Rokne, One-dimensional Thermal Shock Problem With Two Relaxation Times. J. Thermal Stresses, 12, 259-279, 1989.

7. J. Ignaczak, A Strong Discontinuity Wave In Thermoelasticity With Relaxation Times, J. Thermal Stresses, 8, 25-40, 1985.

8. H. H. Sherief and M. N. Anwar, Problem In Generalized Thermoelasticity, J. Thermal Stresses, 9, 165-181, 1986.

9. D. S. Chandrasekharaiah and H. N. Murthy, Temperature-rate-dependent Thermoelastic Interactions Due To A Line Heat Source, Acta Mechanica. 89, $1-12,1991$.

10. A. E. Green and P. M. Naghdi, A Re-xamination of The Basic Postulates of Thermomechanics, Proc. Roy. Soc. London Ser. A, 432, 171-194, 1991.

11. A. E. Green and P. M. Naghdi, Thermoelasticity Without Energy Dissipation, J. Elasticity, 31, 189-208, 1993.

12. A. E. Green and P. M. Naghdi, On Undamped Heat Waves In An Elastic Solid, J. Thermal Stresses, 15, 253-264, 1992.

13. A. Erdelyi, Tables Of Integral Transforms, Vol.1, 2, McGraw-Hill, 1954. 


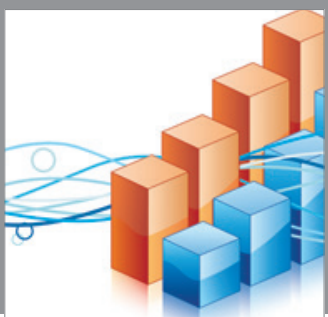

Advances in

Operations Research

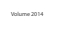

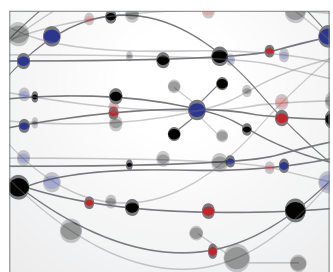

\section{The Scientific} World Journal
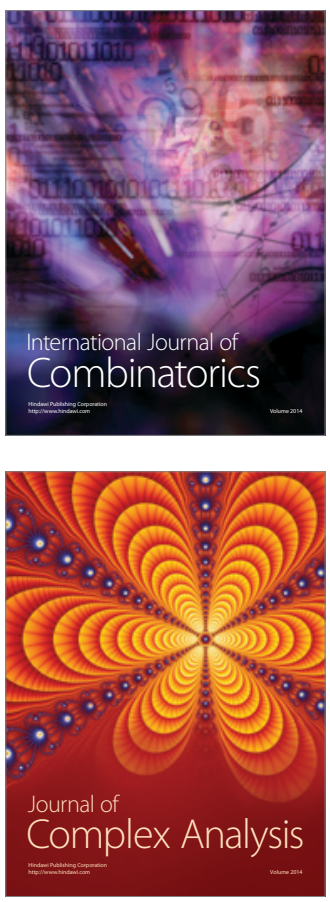

International Journal of

Mathematics and

Mathematical

Sciences
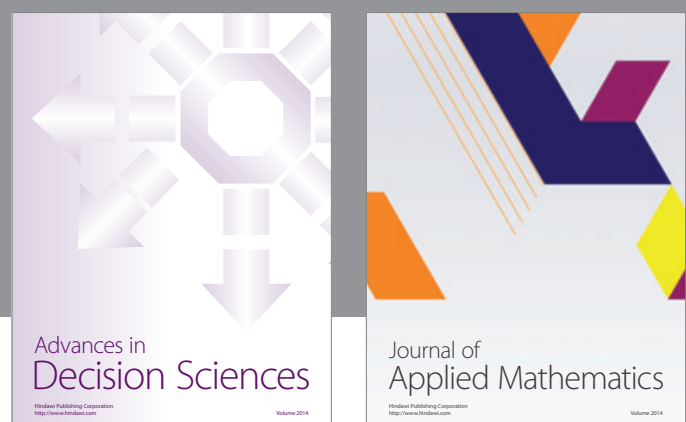

Journal of

Applied Mathematics
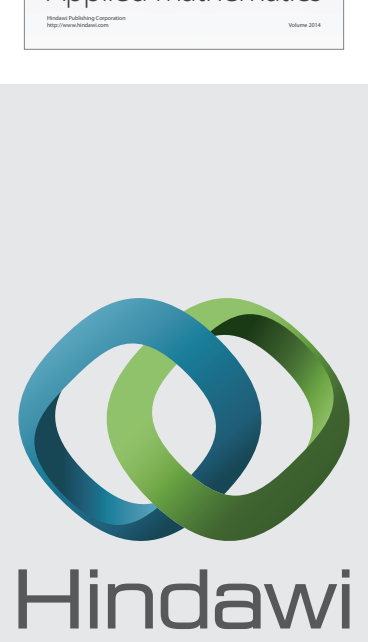

Submit your manuscripts at http://www.hindawi.com
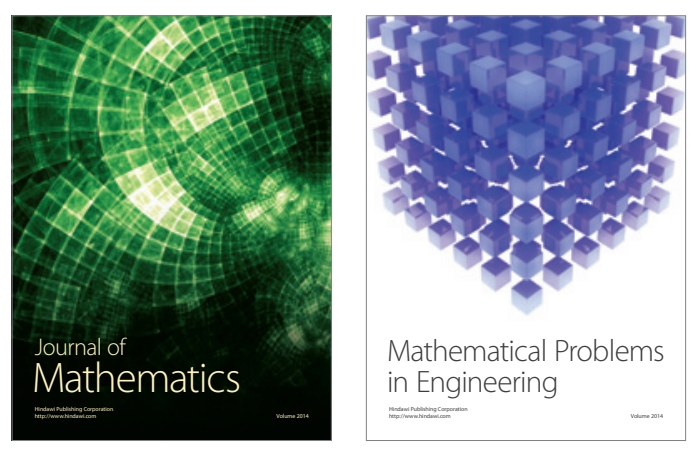

Mathematical Problems in Engineering
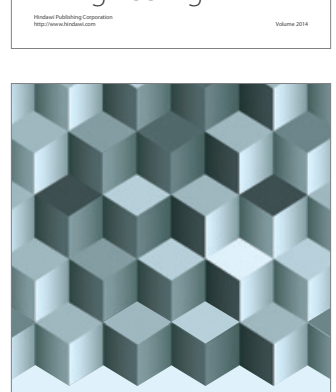

Journal of

Function Spaces
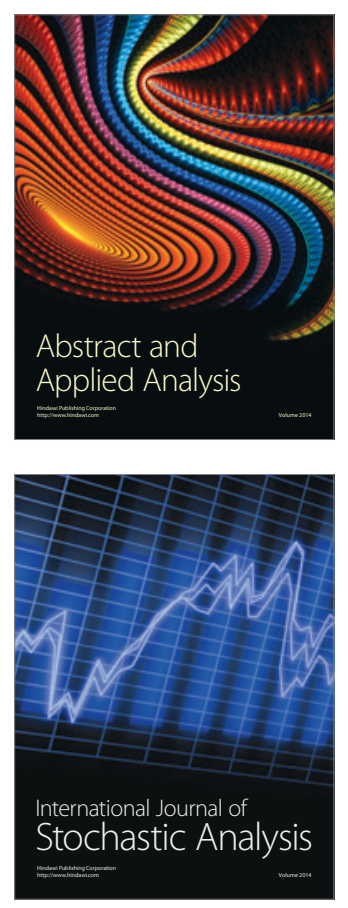

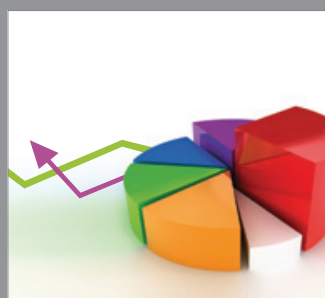

ournal of

Probability and Statistics

Promensencen
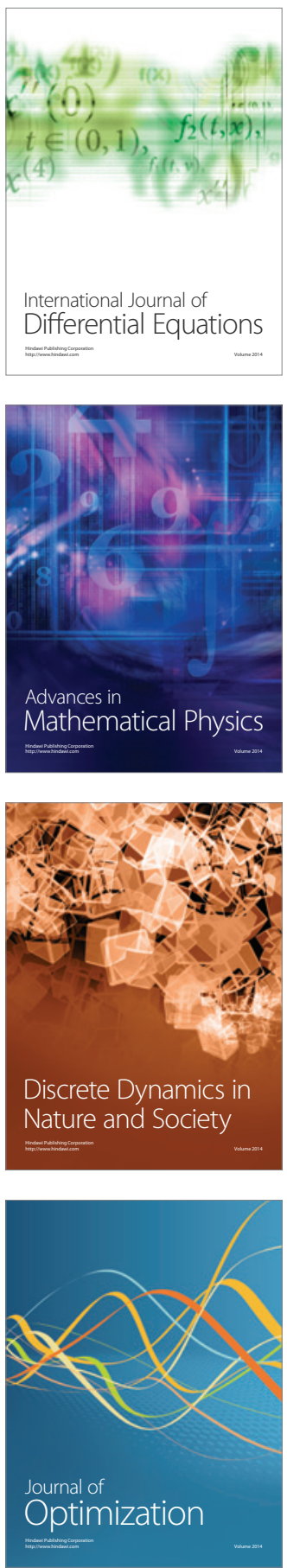